\title{
Algorithm for Finding Feedback in a Problem with Constraints for One Class of Nonlinear Control Systems
}

\author{
M. G. Dmitriev ${ }^{1}$, Z. N. Murzabekov², G. A. Mirzakhmedova ${ }^{2}$
}

DOI: $10.18255 / 1818-1015-2021-3-220-233$

${ }^{1}$ Federal Research Center "Computer Science and Control" of Russian Academy of Sciences, 44/2, Vavilova str., Moscow 119333, Russia.

${ }^{2} \mathrm{Al}$ - Farabi Kazakh National University, 71 al-Farabi Ave., Almaty 050040, Republic of Kazakhstan.

MSC2020: 49J15

Research article

Full text in Russian
Received August 20, 2021

After revision August 31, 2021

Accepted September 1, 2021

For a continuous nonlinear control system on a finite time interval with control constraints, where the right-hand side of the dynamics equations is linear in control and linearizable in the vicinity of the zero equilibrium position, we consider the construction of a feedback according to the Kalman algorithm. For this, the solution of an auxiliary optimal control problem with a quadratic functional is used by analogy with the SDRE approach.

Since this approach is used in the literature to find suboptimal synthesis in optimal control problems with a quadratic functional with formally linear systems, where all coefficient matrices in differential equations and criteria can contain state variables, then on a finite time interval it becomes necessary to solve a complicated matrix differential Riccati equations, with state-dependent coefficient matrices. This circumstance, due to the nonlinearity of the system, in comparison with the Kalman algorithm for linear-quadratic problems, significantly increases the number of calculations for obtaining the coefficients of the gain matrix in the feedback and for obtaining synthesis with a given accuracy. The proposed synthesis construction algorithm is constructed using the extension principle proposed by V. F. Krotov and developed by V. I. Gurman and allows not only to expand the scope of the SDRE approach to nonlinear control problems with control constraints in the form of closed inequalities, but also to propose a more efficient computational algorithm for finding the matrix of feedback gains in control problems on a finite interval. The article establishes the correctness of the application of the extension principle by introducing analogs of the Lagrange multipliers, depending on the state and time, and also derives a formula for the suboptimal value of the quality criterion. The presented theoretical results are illustrated by calculating suboptimal feedbacks in the problems of managing three-sector economic systems.

Keywords: optimal control problem; Lagrange multiplier method; nonlinear system; quadratic functional; feedback; SDRE approach; three-sector economic control object

\section{INFORMATION ABOUT THE AUTHORS}

\author{
Michail G Dmitriev | orcid.org/0000-0002-1184-129X. E-mail: mdmitriev@mail.ru \\ correspondence author Doctor of Sciences, Professor, Chief Researcher. \\ Zainelkhriet N Murzabekov | orcid.org/0000-0002-9074-4753. E-mail: murzabekov-zein@mail.ru \\ Doctor of Sciences, Professor, Chief Researcher. \\ Gulbanu A Mirzakhmedova | orcid.org/0000-0001-7915-945X. E-mail: gulbanu.myrzahmedova@mail.ru \\ Research Fellow, Master of science, Senior Lecturer.
}

Funding: The research was carried out with partial support of the Russian Science Foundation, grant No. 21-11-00202.

For citation: M. G. Dmitriev, Z. N. Murzabekov, and G. A. Mirzakhmedova, "Algorithm for Finding Feedback in a Problem with Constraints for One Class of Nonlinear Control Systems”, Modeling and analysis of information systems, vol. 28, no. 3, pp. 220-233, 2021. 


\section{Алгоритм нахождения обратной связи в задаче с ограничениями для одного класса нелинейных управляемых систем}

М. Г. Дмитриев ${ }^{1}, 3$. Н. Мурзабеков ${ }^{2}$, Г. А. Мирзахмедова ${ }^{2}$

DOI: $10.18255 / 1818-1015-2021-3-220-233$

${ }_{1}^{1}$ Федеральный исследовательский центр “Информатика и управление” Российской академии наук, ул. Вавилова, д. 44/2, г. Москва, 119333 Россия.

${ }^{2}$ Казахский национальный университет имени аль-Фараби, проспект аль-Фараби, д. 71, г. Алматы, 050040 Казахстан.

УДК 517.977 .55

Научная статья

Полный текст на русском языке
Получена 20 августа 2021 г.

После доработки 31 августа 2021 г. Принята к публикации 1 сентября 2021 г.

Для непрерывной нелинейной управляемой системы на конечном интервале времени с ограничениями на управление, где правая часть уравнений динамики линейна по управлению и линеаризуема в окрестности нулевого положения равновесия рассматривается построение обратной связи по схеме алгоритма Калмана. Для этого используется решение вспомогательной задачи оптимального управления с квадратичным функционалом по аналогии с подходом SDRE.

Так как этот подход в литературе применяется для нахождения субоптимального синтеза в задачах оптимального управления с квадратичным функционалом с формально линейными системами, где все матрицы коэффициентов в дифференциальных уравнениях и в критерии могут содержать переменные состояния, то на конечном интервале времени здесь появляется необходимость решения усложненного матричного дифференциального уравнения Риккати, с матрицами коэффициентов зависящими от состояния. Это обстоятельство вследствие нелинейности системы, по сравнению с алгоритмом Калмана для линейно-квадратичных задач, значительно увеличивает количество вычислений для получения коэффициентов матрицы коэффициентов усиления в обратной связи и для получения синтеза с заданной точностью. Предложенный в работе алгоритм построения синтеза строится с помощью принципа расширения, предложенного В.Ф. Кротовым и развитого В.И. Гурманом, и позволяет не только расширить сферу использования подхода SDRE на нелинейные задачи управления с ограничениями на управление в виде замкнутых неравенств, но и предложить более эффективный вычислительный алгоритм нахождения матрицы коэффициентов усиления обратной связи в задачах управления на конечном интервале. В работе устанавливается корректность применения принципа расширения с помощью введения аналогов множителей Лагранжа, зависящих от состояния и времени, а также выводится формула субоптимального значения критерия качества. Приведенные теоретические результаты иллюстрируются на расчетах субоптимальных обратных связей в задачах управления трехсекторными экономическими системами.

Ключевые слова: задача оптимального управления; метод множителей Лагранжа; нелинейная система; квадратичный функционал; обратная связь; подход SDRE; трехсекторный экономический объект управления

\section{ИНФОРМАЦИЯ ОБ АВТОРАХ}

Михаил Геннадьевич Дмитриев автор для корреспонденции

Зайнелхриет Нугманович Мурзабеков

Гулбану Абсаматовна Мирзахмедова
orcid.org/0000-0002-1184-129X. E-mail: mdmitriev@mail.ru

ГНС, доктор физ.-мат. наук, профессор.

orcid.org/0000-0002-9074-4753. E-mail: murzabekov-zein@mail.ru ГНС, доктор технических наук, профессор.

orcid.org/0000-0001-7915-945X. E-mail: gulbanu.myrzahmedova@mail.ru НС, магистр, старший преподаватель.

Финансирование: Исследование выполнено при частичной поддержке гранта РНФ № 21-11-00202.

Для цитирования: M. G. Dmitriev, Z. N. Murzabekov, and G. A. Mirzakhmedova, “Algorithm for Finding Feedback in a Problem with Constraints for One Class of Nonlinear Control Systems”, Modeling and analysis of information systems, vol. 28, no. 3, pp. 220-233, 2021.

(ㄷ Дмитриев М. Г., Мурзабеков 3.Н., Мирзахмедова Г.А., 2021

Эта статья открытого доступа под лицензией СС BY license (https://creativecommons.org/licenses/by/4.0/). 


\section{Введение}

Задачи и вопросы техники построения синтезирующих законов управления продолжают быть актуальными, в связи с необходимостью построения законов обратной связи в различных приложениях. Это происходит на фоне усложняющихся математических моделей из-за необходимости учета нелинейностей, возмущений, повышений размерности векторов состояния и управления и т. д. В связи с этим происходит постоянный поиск новых подходов к построению синтезирующих законов управления в нелинейных задачах и развитие имеющихся способов построения законов обратной связи в динамических системах. При этом наличие ограничений, конечно, осложняет поиск таких алгоритмов управления. Для приближенного решения задач синтеза в нелинейном случае с 90-х годов прошлого века в литературе активно развивается так называемый подход SDRE (cм., например, [1-6]) для задач оптимального управления в классической постановке без ограничений на управление. Для приближенного решения нелинейных задач оптимального управления как на бесконечном интервале, так и на конечном, здесь правые части обыкновенных дифференциальных уравнений динамики сначала приводятся к формально линейному виду по состоянию и управлению, где коэффициенты всех матриц могут быть зависящими от состояния. Обратная связь, при этом, строится с помощью решения соответствующих линейно-квадратичных задач оптимального управления, где коэффициенты весовых матриц в критерии оптимальности также могут зависеть от переменных состояния. Затем матрица коэффициентов усиления регулятора находится с помощью решения матричных уравнений типа Риккати, как алгебраических для задач стабилизации на полуоси, так и дифференциальных, для задач управления на конечном интервале времени соответственно. Как показали многочисленные эксперименты, такой эвристический подход вследствие неоднозначности представления нелинейной системы в виде системы линейной структуры и погрешностей, возникающих при численном решении матричных уравнений Риккати, коэффициенты также зависят от состояния, порождает множество возможных субоптимальных решений. Но учитывая сложность построения и важность для приложений управления в виде законов обратной связи в нелинейных системах, подход SDRE получил широкое распространение в литературе при приближенном решении нелинейных задач оптимального управления без ограничений на управление.

Здесь, для одного класса нелинейных управляемых систем на конечном интервале времени, показывается возможность обоснования подхода SDRE при построении обратной связи в задачах с ограничениями на управление с помощью принципа расширения, предложенного В. Ф. Кротовым [7] и развитым В. И. Гурманом [8]. При этом, предлагается более эффективный алгоритм с точки зрения объема вычислений, не требующий многократного интегрирования матричных дифференциальных уравнений Риккати с коэффициентами, зависящими от состояния. Отметим, что впервые применение принципа расширения Кротова в рамках подхода SDRE, иллюстрировалось в [9] для задачи построения стабилизирующего регулятора без ограничений на управление на бесконечном интервале времени.

В конце настоящей работы приводятся результаты вычислительного эксперимента, иллюстрирующие предлагаемый алгоритм построения обратной связи на примере решения задачи оптимального управления для трехсекторной нелинейной модели экономического объекта, с ограничениями на управление в виде замкнутых неравенств.

\section{1. Теоретические результаты}

Пусть нелинейная управляемая система имеет вид

$$
\dot{y}(t)=A y(t)+B(y) u(t)+h(y), \quad y\left(t_{0}\right)=y_{0}, \quad t \in\left[t_{0}, T\right],
$$

где $y(t) \in R^{n}, u(t) \in R^{m}$ вектор-функции состояния и управления, $n \times n$ матрица А - постоянная, коэффициенты $n \times m$ матрицы $B(y)$ и компоненты вектора $h(y)$ ограничены и непрерывно диффе- 
ренцируемы по $y(t) \in R^{n}$, а управление $u \in R^{m}$ - кусочно-непрерывная вектор-функция; $t_{0}, T$ заданные начальный и конечный момент времени, управление $u(t)$ в каждый момент времени, удовлетворяет ограничениям

$$
u(t) \in U(t)=\left\{u: \gamma_{1}(t) \leq u(t) \leq \gamma_{2}(t), t \in\left[t_{0}, T\right] ; \quad \gamma_{1}, \gamma_{2} \in C^{m}\left[t_{0}, T\right]\right\} .
$$

Вид (1) связан с рассмотрением нелинейных задач управления, когда исходная система уравнений динамики может иметь ненулевое положение равновесия $x^{s}$ при некотором постоянном управлении $v^{s}$, а неоднородность $h(y)$ связана с преобразованием $y=x-x^{s}$ и выделением линейной части по $y$, т.е. $h(0)=0$.

Будем искать управление, на основе подхода SDRE, используя критерий оптимальности

$$
J(u)=\frac{1}{2} y^{\prime}(T) F y(t)+\frac{1}{2} \int_{t_{0}}^{T}\left(y^{\prime} Q(y) y+u^{\prime} R u\right) d t,
$$

где матрица $Q$ предполагается положительно полуопределенной при всех допустимых $y$, а матрицы $F, R$ постоянные и положительно определенные.

Теперь задача состоит в том, чтобы найти управление $u(t)$ в виде обратной связи путем подбора матрицы $Q(y)$ так, чтобы достигался минимум критерия (3) вдоль траекторий системы (1) при выполнении ограничений (2). Такой подход связан, с одной стороны, со стремлением, чтобы вдоль такого управления траектории замкнутой системы, как минимум, оставались ограниченными, а, с другой стороны, являлись бы рациональными в некотором смысле.

Заметим, что весовая матрица $Q(y)$ в критерии будет представляться в виде

$$
Q(y)=\left(K+W^{-1}\right) B(y) R^{-1} B^{\prime}(y)\left(K+W^{-1}\right)-\left(K+W^{-1}\right) B_{s} R^{-1} B_{s}^{\prime}\left(K+W^{-1}\right)+Q_{1},
$$

где $B_{s}=B(0), Q_{1}$ - некоторая положительно определенная постоянная матрица и будет подбираться так, чтобы матрица $Q(y)$ при всех $y$ была бы положительно полуопределенной, матрица $K$ являлась бы решением некоторого матричного алгебраического уравнения Риккати с постоянными коэффициентами, а невырожденная матрица $W$ будет определяться ниже.

Итак, будем искать оптимальное управление в форме обратной связи $u(y, t)$ в задаче (1)-(3). Для решения используем принцип расширения [7,8], который состоит в том, что исходная задача оптимального управления с ограничениями сводится к задаче без ограничений и при этом решение новой задачи является одновременно и решением первоначальной задачи [9-13]. Для этого задачу (1)-(3) заменяем задачей без ограничений с использованием множителей Лагранжа $\lambda_{1}(t), \lambda_{2}(t), \lambda_{3}(t), \mu(y, t)$. Неотрицательные функции $\lambda_{1}(t), \lambda_{2}(t)$ отвечают ограничениям на управление, множитель Лагранжа $\mu(y, t)$ вводится для учета дифференциальных связей в (1) и ищется в виде $\mu(y, t)=K y+q(y, t)$, а функция $\lambda_{3}(t)$ отвечает вводимой связи типа равенства $y(t)-W(t) q(y, t)=0$.

Итак, функционал (3) по схеме $[7,8]$ заменяем следующим

$$
\begin{array}{r}
L(y, u)=\frac{1}{2} y^{\prime}(T) F y(T)+\int_{t_{0}}^{T}\left\{\frac{1}{2} y^{\prime} Q(y) y+u^{\prime} R u+\mu^{\prime}(y, t)(A y+B(y) u+h(y)-\right. \\
\left.\left.-\dot{y})+\lambda_{1}^{\prime}\left[\gamma_{1}-u\right]+\lambda_{2}^{\prime}\left[u-\gamma_{2}\right]+\lambda_{3}^{\prime}[y-W(t) q)\right]\right\} d t,
\end{array}
$$

и введем функции

$$
\begin{array}{r}
M(y, u, t)=\frac{1}{2} y^{\prime} Q(y) y+\frac{1}{2} u^{\prime} R u+(K y+q)^{\prime}(A y+B(y) u+ \\
+h(y))+y^{\prime} \dot{q}+\lambda_{1}^{\prime}\left[\gamma_{1}-u\right]+\lambda_{2}^{\prime}\left[u-\gamma_{2}\right]+\lambda_{3}^{\prime}[y-W(t) q],
\end{array}
$$




$$
\begin{gathered}
G\left(y_{0}, y(T)\right)=\frac{1}{2} y^{\prime}(T)(F-K) y(T)-y^{\prime}(T) q(T)+\frac{1}{2} y\left(t_{0}\right)^{\prime} K y\left(t_{0}\right)+y\left(t_{0}\right)^{\prime} q\left(t_{0}\right), \\
v(y, t)=\frac{1}{2} y^{\prime} K y+y^{\prime} q(y, t) .
\end{gathered}
$$

Перепишем (5) в виде

$$
L(y, u)=\frac{1}{2} y^{\prime}(T) F y(T)+\left[v\left(y_{0}, t_{0}\right)-v(y(T), T)\right]+\int_{t_{0}}^{T} M(y, u, t) d t
$$

Введем множество всех допустимых управлений, удовлетворяющих условию $u(t) \in U(t), t \in$ $\left[t_{0}, T\right]$ и соответствующих им траекторий $y(t)$ системы (1), определенных на отрезке $t_{0} \leq t \leq T$, т. е. множество всех допустимых пар $\{y(t), u(t)\}$, которое обозначим

$$
\Delta\left(t_{0}, T, y_{0}\right)=\left\{(y, u): u(t) \in U(t), \dot{y}(t)=A y+B(y) u(t)+h(y), y\left(t_{0}\right)=y_{0}, t_{0} \leq t \leq T\right\} .
$$

Учитывая (7), $L(y, u)$ принимает вид

$$
L(y, u)=G\left(y_{0}, y(T)\right)+\int_{t_{0}}^{T} M(y(t), u(t), t) d t
$$

где исходная задача с ограничениями сведена к другой задаче, но уже без ограничений.

Теперь обратимся к непрерывно дифференцируемой функции $v(y, t)$ в $(8)$, и вычислим ее полную производную по времени $\frac{d v}{d t}=\left(\frac{\partial v}{\partial y}\right)^{\prime} \dot{y}+\frac{\partial v}{\partial t}$.

Ниже будем использовать обозначения $M_{\min } \equiv \inf \left\{M(y, u, t),\{y, u\} \in \Delta\left(t_{0}, T, y_{0}\right)\right\}$, $G_{\text {min }} \equiv \inf \left\{G\left(y_{0}, y(T)\right),\left\{y_{0}, y(T)\right\} \in \Delta\left(t_{0}, T, y_{0}\right)\right\}$ при почти всех $t \in\left[t_{0}, T\right]$.

Имеет место

Лемма. Пусть выполнены условия:

1) Пара $\{y(t), u(t)\} \in \Delta\left(t_{0}, T, y_{0}\right)$;

2) Существуют $\lambda_{1}(t) \geq 0, \lambda_{2}(t) \geq 0$ такие, что на оптимальной паре $(\tilde{y}(t), \tilde{u}(t)) \in \Delta\left(t_{0}, T, y_{0}\right)$, которая доставляет минимальное значение функционалу (11), выполняются условия

$$
\lambda_{1}^{\prime}\left(\gamma_{1}-\tilde{u}\right)=0, \lambda_{2}^{\prime}\left(\tilde{u}-\gamma_{2}\right)=0 .
$$

Тогда справедливы следующие соотношения

$$
L(y, u)=\int_{t_{0}}^{T} M(y(t), u(t), t) d t+G\left(y_{0}, y(T)\right) \leq J(u), \quad L(\tilde{y}, \tilde{u})=J(\tilde{u}),
$$

где $(y(t), u(t)),(\tilde{y}(t), \tilde{u}(t))$ допустимая и оптимальная пары соответственно.

Доказательство. Для функции $v(y, t)$ из (8) имеем

$$
\frac{d v(y(t), t)}{d t}=\frac{\partial v(y(t), t)}{\partial y} \dot{y}+\frac{\partial v(y(t), t)}{\partial t}=(K y+q)^{\prime} \dot{y}+y^{\prime} \frac{d q(y(t), t)}{d t}
$$

Интегрируя (14) и суммируя полученное выражение с функционалом (5), получаем

$$
\begin{aligned}
L(y, u) & =\int_{t_{0}}^{T}\left\{\frac{1}{2} y^{\prime} Q(y) y+u^{\prime} R u+(K y+q)^{\prime}(A y+B(y) u+h(y))+y^{\prime} \dot{q}-\right. \\
& \left.\left.-\frac{d v}{d t}+\lambda_{1}^{\prime}\left[\gamma_{1}-u\right]+\lambda_{2}^{\prime}\left[u-\gamma_{2}\right]+\lambda_{3}^{\prime}[y-W(t) q)\right]\right\} d t+\frac{1}{2} y^{\prime} F y(T)
\end{aligned}
$$


Отсюда имеем, с учетом (6) и (7), что $L(y, u)=\int_{t_{0}}^{T} M(y(t), u(t), t) d t+G\left(y_{0}, y(T)\right)$ и

$$
G\left(y_{0}, y(T)\right)=\frac{1}{2} y^{\prime}(T)(F-K) y(T)-y^{\prime}(T) q(T)+\frac{1}{2} y^{\prime}\left(t_{0}\right) K y\left(t_{0}\right)+y^{\prime}\left(t_{0}\right) q\left(t_{0}\right),
$$

Покажем, что на допустимых решениях выполняется неравенство $L(y(t), u(t)) \leq J(u)$. Действительно, пусть $(y(t), u(t)) \in \Delta\left(t_{0}, T, y_{0}\right)$, тогда в силу (1) из (5), учитывая $\lambda_{1}^{\prime}\left(\gamma_{1}-u\right) \leq 0, \lambda_{2}^{\prime}\left(u-\gamma_{2}\right) \leq$ $0, \lambda_{3}^{\prime}[y-W(t) q]=0$, получаем

$$
\begin{array}{r}
L(y, u)=\int_{t_{0}}^{T}\left\{\frac{1}{2} y^{\prime} Q(y) y+\frac{1}{2} u^{\prime} R u+(K y+q)^{\prime}(A y+B(y) u+h(y)-\right. \\
\left.\left.-\dot{y})+\lambda_{1}^{\prime}\left[\gamma_{1}-u\right]+\lambda_{2}^{\prime}\left[u-\gamma_{2}\right]+\lambda_{3}^{\prime}[y-W(t) q)\right]\right\} d t+\frac{1}{2} y^{\prime}(T) F y(T)= \\
\left.=J(u)+\int_{t_{0}}^{T}\left\{\lambda_{1}^{\prime}\left[\gamma_{1}-u\right]+\lambda_{2}^{\prime}\left[u-\gamma_{2}\right]+\lambda_{3}^{\prime}[y-W(t) q)\right]\right\} d t \leq J(u) .
\end{array}
$$

Из цепочки рассуждений в (15) видно, что вдоль оптимальной пары $(\tilde{y}(t), \tilde{u}(t)) \in \Delta\left(t_{0}, T, y_{0}\right)$, учитывая (12), выполняется следующее равенство

$$
L(\tilde{y}(t), \tilde{u}(t))=\int_{t_{0}}^{T}\left[\lambda_{1}^{\prime}\left(\gamma_{1}-u\right)+\lambda_{2}^{\prime}\left(u-\gamma_{2}\right)\right] d t+J(\tilde{u})=J(\tilde{u})
$$

и, таким образом, утверждение леммы имеет место.

Для определения пары $\{\tilde{y}(t), \tilde{u}(t)\}$, минимизирующей функционал (11), необходимо найти управление $u(t)$ и определить множители $\mu(y, t)=K y+q(y, t), \lambda_{1}(t), \lambda_{2}(t)$ так, чтобы при каждом фиксированном $t \in\left(t_{0}, T\right)$ подынтегральная функция $M(y, u, t)$ в (11) достигала наименьшего значения среди $(y, u) \in \Delta\left(t_{0}, T, y_{0}\right)$.

Учитывая, что при $t=T$ функция $G\left(y_{0}, y(T)\right)$ принимает минимальное значение, а пара $\{\tilde{y}(t), \tilde{u}(t)\}$ удовлетворяет (1) при выполнении ограничений (2), тогда из необходимых условий минимума по управлению для функции $M(y, u, t)$ получаем выражение для управления

$$
u=-R^{-1} B^{\prime}(y)(K y+q)-R^{-1}\left(\lambda_{2}-\lambda_{1}\right),
$$

а из минимизации терминальной части (7) функционала (11) находим $\left.q\right|_{t=T}=(F-K) y(T)$.

Теперь определим неизвестные матрицы $K, W(t)$ и вектор-функцию $q$ как решения на отрезке $t \in\left[t_{0}, T\right]$ следующих уравнений

$$
\begin{gathered}
K A+A^{\prime} K-K B_{s} R^{-1} B_{s}^{\prime} K+Q_{1}=0, \\
\dot{W}=W A_{1, s}^{\prime}+A_{1, s} W-B_{1, s}, \quad W(T)=(F-K)^{-1}, \\
\dot{q}=-\left(A-B_{s} R^{-1} B_{s}^{\prime} K\right)^{\prime} q+W^{-1}(t)\left(h(y)-\left(B_{1}(y)-B_{1, s}\right)(K y+q)+B(y) \varphi(y, t)\right), \\
q(y, T)=W^{-1}(T) y(T),
\end{gathered}
$$

где постоянные матрицы $A_{1, s}, B_{1, s}$ имеют вид $A_{1, s}=A-B_{s} R^{-1} B_{s}^{\prime} K, \quad B_{1, s}=B_{s} R^{-1} B_{s}^{\prime}$, и предполагается, что $K$ положительно определенная матрица, матрица $W(t)$ невырожденная, постоянная матрица $F$ такая, что $F>>$, а под $\dot{q}$ понимается полная производная по времени.

Покажем, что если существуют решения уравнений (17), (18), тогда замкнутая система вдоль управления (16) принимает вид

$$
\dot{y}=A_{1}(y) y-B_{1}(y) q+B(y) \varphi(y, t)+h(y), \quad y\left(t_{0}\right)=y_{0},
$$


где

$$
\begin{array}{r}
A_{1}(y)=A-B(y) R^{-1} B(y)^{\prime} K, \quad B_{1}(y)=B(y) R^{-1} B(y)^{\prime}, \quad \varphi(y, t)=R^{-1}\left[\lambda_{1}(y, t)-\lambda_{2}(y, t)\right] \\
\lambda_{1}(y, t)=R \max \left\{0 ; \gamma_{1}-\omega(y, t)\right\} \geq 0, \quad \lambda_{2}(y, t)=R \max \left\{0 ; \omega(y, t)-\gamma_{2}\right\} \geq 0, \\
\omega(y, t)=-R^{-1} B^{\prime}(y)(K y+q)
\end{array}
$$

Действительно, т.к. $q=q(y, t),\left.q\right|_{t=T}=(F-K) y(T)$ и мы ее ввели как функцию $q(y, t)=W^{-1}(t) y(t)$ или $y \equiv W q$, то вычисляя теперь $\dot{y} \equiv \dot{W} q+W \dot{q}$ и учитывая уравнения (17)-(19), получаем

$$
A_{1}(y) y(t)-B_{1}(y) q(t)+B(y) \varphi(y, t)+h(y)=\left(W A_{1, s}^{\prime}+A_{1, s} W-B_{1, s}\right) q+W \dot{q}
$$

После преобразования левой части, используя уравнения в (17), (18) имеем

$$
\begin{array}{r}
\left(A_{1}(y)-A_{1, s}\right) W q+A_{1, s} W q-\left(B_{1}(y)-B_{1, s}\right) q-B_{1, s} q+B(y) \varphi(y, t)+h(y)= \\
=\left(W A_{1, s}^{\prime}+A_{1, s} W-B_{1, s}\right) q+W \dot{q}, \\
-\left(B_{1}(y)-B_{1, s}\right)(K y+q)+B(y) \varphi(y, t)+h(y)=W A_{1, s}^{\prime}+W \dot{q},
\end{array}
$$

Отсюда находим, что

$$
\begin{array}{r}
\dot{q}=-\left(A_{1, s}\right)^{\prime} q+W^{-1}(t)\left(h(y)-\left(B_{1}(y)-B_{1, s}\right)(K y+q)+B(y) \varphi(y, t)\right) \\
\left.q\right|_{t=t_{0}}=W^{-1}\left(t_{0}\right) y\left(t_{0}\right)
\end{array}
$$

Результаты, установленные для задачи (1)-(3), сформулируем в виде следующего утверждения.

Теорема 1. Пусть выполняются условия леммы и дополнительно предположим, что:

1. Существует постоянная положительно определенная матрица $Q_{1}$, что матрица $Q(y)$ при всех y является положительно полуопределенной.

2. Пара постоянных матриц $\left\{A, B_{s}\right\}$ удовлетворяет условию управляемости.

3. Постоянная положительно определенная матрица $F$ такая, что $F-K$ также является положительно определенной матрицей.

4. Функция $q(y, t)$ удовлетворяет соотношению (22).

Тогда

а) существует положительно определенное решение матричного алгебраического уравнения Риккати (17);

b) решение $W(t)$ дифференциального уравнения (18) существует единственно и является невырожденной матрицей при всех $t \in\left[t_{0}, T\right]$;

с) оптимальное управление $u(y, t)$ в задаче (1)-(3) имеет вид

$$
u(y, t)=-R^{-1} B^{\prime}(y)(K y+q(y, t))+\varphi(y, t)
$$

где $q(y, t), \varphi(y, t)$ определяется в (19), (21).

Доказательство. Учитывая утверждения леммы, вследствие первых двух условий теоремы, подынтегральное выражение в (3) неотрицательное при всех допустимых парах ( $y, u)$. Поэтому из необходимых условий оптимальности $\frac{\partial M}{\partial u}=0$ имеем, что экстремальное управление представляется как $u=-R^{-1}\left[B^{\prime}(y)(K y+q)-\lambda_{1}+\lambda_{2}\right]$, которое с учетом обозначений в (21) принимает вид (23). Далее определяем множители $\lambda_{1} \geq 0, \lambda_{2} \geq 0$ таким образом, чтобы, с одной стороны, выполнялись условия

$$
\lambda_{1}^{\prime}\left(\gamma_{1}-\tilde{u}\right)=0, \quad \lambda_{2}^{\prime}\left(\tilde{u}-\gamma_{2}\right)=0
$$


а с другой, осуществляем выбор $\lambda_{1}, \lambda_{2}, \varphi$ так, что имеют место представления

$$
\lambda_{1}(y, t)=-\operatorname{Rinf}\left(0, \omega(y, t)-\gamma_{1}(t)\right), \quad \lambda_{2}(y, t)=-\operatorname{Rinf}\left(0, \gamma_{2}(t)-\omega(y, t)\right)
$$

и

$$
\varphi(y, t)=-\inf \left(0, \omega(y, t)-\gamma_{1}(t)\right)+\inf \left(0, \gamma_{2}(t)-\omega(y, t)\right) .
$$

Теперь определим функцию $M(y, u, t)$ на управлении (23). Подставляя управление (23) в выражение (6) и группируя подобные члены, в результате получим функцию

$$
\begin{array}{r}
P(y, t)=\frac{1}{2} y^{\prime} Q(y) y+(K y+q)^{\prime} A y-\frac{1}{2}(K y+q)^{\prime} B(y) R^{-1} B^{\prime}(y)(K y+q)+ \\
+\frac{1}{2} \varphi^{\prime} R \varphi+(K y+q)^{\prime} h(y)+y^{\prime} \dot{q}=\frac{1}{2} y^{\prime}\left[Q_{1}+K A+A^{\prime} K-K B_{s} R^{-1} B_{s}^{\prime} K\right] y+ \\
+y^{\prime}\left(A_{1 s}^{\prime} q+\dot{q}\right)+\frac{1}{2} \varphi^{\prime} R \varphi+(K y+q)^{\prime} h(y)-\frac{1}{2} q^{\prime} B_{1 s} q .
\end{array}
$$

Тогда вдоль положительно определенной матрицы $K$ из (17) и функции $q(t, y)$ из $(22)$, для $\{\tilde{y}(t), \tilde{u}(t)\} \in \Delta\left(t_{0}, T, y_{0}\right)$ имеем

$$
L(\tilde{y}, \tilde{u})=\int_{t_{0}}^{T}\left\{y^{\prime}\left(A_{1 s}^{\prime} q+\dot{q}\right)+\frac{1}{2} \varphi^{\prime} R \varphi+(K y+q)^{\prime} h(y)-\frac{1}{2} q^{\prime} B_{1 s} q\right\} d t+G\left(y_{0}, \tilde{y}(T)\right),
$$

где в точке $t=T$ функция $G\left(y_{0}, \tilde{y}(T)\right)$ в (7) также принимает минимальное значение, что определяет минимальное значение (27) в целом.

Итак, имеем

$$
\begin{gathered}
M(\tilde{y}(t), \tilde{u}(t), t)=q^{\prime}\left(h(y)-\left(B_{1}(y)-B_{1 s}\right)(K y+q)+B(y) \varphi(y, t)\right)+\frac{1}{2} \varphi^{\prime} R \varphi+(K y+q)^{\prime} h(y)-\frac{1}{2} q^{\prime} B_{1 s} q, \\
G\left(y_{0}, \tilde{y}(T)\right)=\frac{1}{2} y^{\prime}(T)(F-K) y(T)+\frac{1}{2} y^{\prime}\left(t_{0}\right) K y\left(t_{0}\right)+y^{\prime}\left(t_{0}\right) q\left(t_{0}\right) .
\end{gathered}
$$

Теперь нетрудно показать, что управление (23) является оптимальным, т. е. вдоль него критерий (3) принимает минимальное значение. Пусть существуют функции $\lambda_{1}(y, t), \lambda_{2}(y, t)$ удовлетворяющие условию теоремы и произвольная допустимая пара $(y(t), u(t)) \in \Delta\left(t_{0}, T, y_{0}\right)$, тогда согласно (24) будем иметь

$$
\begin{array}{r}
J(u)-J(\tilde{u}) \geq L(y(t), u(t))-L(\tilde{y}(t), \tilde{u}(t))= \\
=\int_{t_{0}}^{T}[M(y(t), u(t), t)-M(\tilde{y}(t), \tilde{u}(t), t)] d t+G\left(y_{0}, y(T)\right)-G\left(y_{0}, \tilde{y}(T)\right)= \\
=\int_{t_{0}}^{T}\left[M(y(t), u(t), t)-M_{\text {min }}\right] d t+G\left(y_{0}, y(T)\right)-G_{\text {min }} \geq 0
\end{array}
$$

отсюда следует $J(\tilde{u})=L(\tilde{y}(t), \tilde{u}(t))=i n f_{\langle x, u\rangle \in \Delta} L(y(t), u(t))$ и, таким образом, теорема доказана.

Замечание 1. Прием в подходе SDRE с подбором $Q_{1}$, чтобы матрица подобная $Q(y)$ была положительно определенной при всех значениях вектора состояния, демонстрировался в [14].

Имеет место

Следствие 1. В условиях теоремы 1 вдоль оптимального управления (23) минимальное значение критерия в задаче (1)-(3) имеет вид

$$
J(\tilde{u})=L_{\min }(y, u)=\int_{t_{0}}^{T}\left[\frac{1}{2} \varphi^{\prime} R \varphi+y^{\prime}\left(K+W^{-1}\right) h(y)\right] d t+\frac{1}{2} y_{0}^{\prime}\left(K+W_{0}^{-1}\right) y_{0} .
$$


Доказательство. В условиях теоремы 1 справедливо выражение (27). Учитывая (23) и (24) вычислим производную от вспомогательной квадратичной формы $\frac{1}{2} q^{\prime} W q$. Имеем

$$
\begin{aligned}
\frac{d}{d t}\left(\frac{1}{2} q^{\prime} W q\right)=q^{\prime} W\left[-\left(A_{1 s}\right)^{\prime} q+W^{-1}(t, T)\left(h(y)-\left(B_{1}(y)-\right.\right.\right. & \left.\left.\left.B_{1 s}\right)(K y+q)+B(y) \varphi(y, t)\right)\right]+ \\
& +\frac{1}{2} q^{\prime}\left[W A_{1 s}^{\prime}+A_{1 s} W-B_{1 s}\right] q
\end{aligned}
$$

и преобразуем минимальное значение функционала (27) следующим образом. Сначала подставим (30) в выражение (27). Группируя члены, получаем

$$
L_{\min }(y, u)=\int_{t_{0}}^{T}\left[\frac{1}{2} \varphi^{\prime} R \varphi+y^{\prime}\left(K+W^{-1}\right) h(y)\right] d t+\frac{1}{2} y_{0}^{\prime}\left(K+W_{0}^{-1}\right) y_{0} .
$$

Теперь преобразуем минимальное значение функционала (3). Для этого в (3) подставляем оптимальное управление (23) и выражение для $Q(y)$ из (4).

$$
\begin{array}{r}
J(\tilde{u})=\int_{t_{0}}^{T}\left[\frac{1}{2} y^{\prime} Q(y) y+\frac{1}{2} \tilde{u}^{\prime} R \tilde{u}\right] d t+\frac{1}{2} y^{\prime}(T) F y(T)= \\
=\int_{t_{0}}^{T}\left[\frac{1}{2} y^{\prime} Q_{1} y-\frac{1}{2}(K y+q)^{\prime} B_{1 s}(K y+q)+(K y+q)^{\prime} B_{1}(y)(K y+q)\right] d t+ \\
+\int_{t_{0}}^{T}\left[-(K y+q)^{\prime} B(y) \varphi+\frac{1}{2} \varphi^{\prime} R \varphi\right] d t+\frac{1}{2} y^{\prime}(T) F y(T) .
\end{array}
$$

Затем, используя дифференциальное уравнение в (20) и алгебраическое матричное уравнение Риккати (17), последнее выражение преобразуется к виду

$$
\begin{array}{r}
J(\tilde{u})=\int_{t_{0}}^{T}\left[\frac{1}{2} y^{\prime} Q_{1} y-\frac{1}{2}(K y+q)^{\prime} B_{1 s}(K y+q)+(K y+q)(-\dot{y}+A y+h(y))+\frac{1}{2} \varphi^{\prime} R \varphi\right] d t+ \\
+\frac{1}{2} y^{\prime}(T) F y(T)=\int_{t_{0}}^{T}\left[y^{\prime} A_{1 s}^{\prime} q-\frac{1}{2} q^{\prime} B_{1 s} q+(K y+q)(-\dot{y}+A y+h(y))+\frac{1}{2} \varphi^{\prime} R \varphi\right] d t+\frac{1}{2} y^{\prime}(T) F y(T) .
\end{array}
$$

Теперь, вычисляя производную от квадратичной формы $\frac{1}{2} y^{\prime}\left(K+W^{-1}\right) y$ с учетом дифференциальных связей в (18), имеем

$$
\begin{array}{r}
\frac{d}{d t}\left(\frac{1}{2} y^{\prime}\left(K+W^{-1}\right) y\right)=y^{\prime}\left(K+W^{-1}\right) \dot{y}-\frac{1}{2} y^{\prime}\left(W^{-1} \dot{W} W^{-1}\right) y= \\
=y^{\prime}\left(K+W^{-1}\right) \dot{y}-\frac{1}{2} q^{\prime}\left[W A_{1 s}^{\prime}+A_{1 s} W-B_{1 s}\right] q,
\end{array}
$$

Далее, преобразуем минимальное значение функционала (32) используя (33) и получаем

$$
\begin{aligned}
J(\tilde{u})=\int_{t_{0}}^{T}\left[(K y+q)^{\prime} h(y)+\frac{1}{2} \varphi^{\prime} R \varphi\right] d t+\frac{1}{2} y^{\prime}(T) F y(T) & -\frac{1}{2} y^{\prime}(T)\left(K+W^{-1}(T)\right) y(T)+ \\
& +\frac{1}{2} y^{\prime}\left(t_{0}\right)\left(K+W^{-1}(T)\right) y\left(t_{0}\right) .
\end{aligned}
$$

Теперь, учитывая представление $q(y, t)=W^{-1}(t) y(t)$ и условие, что $\left.q\right|_{t=T}=(F-K) y(T)$ получаем, что формула (29)

$$
J(\tilde{u})=\frac{1}{2} y_{0}^{\prime}\left(K+W^{-1}\left(t_{0}\right)\right) y_{0}+\int_{t_{0}}^{T}\left[\frac{1}{2} \varphi^{\prime} R \varphi+y^{\prime}\left(K+W^{-1}(t)\right) h(y)\right] d t
$$


имеет место.

Замечание 2. Отметим, что формула (29) включает в себя известное представление начального условия для функции Беллмана в задаче оптимальной стабилизации для стационарной линейно квадратичной задачи на полуоси без ограничений на управление, и в ней еще, естественно, отражается возрастание минимального значения из-за наличия ограничений на управление, а также учитывается влияние неоднородности $h(y)$.

Опишем алгоритм решения задачи оптимального управления (1)-(3).

1. Находится положительно определенная матрица $Q_{1}$ такая, что $Q(y)$ также будет положительно определенной матрицей при всех $y$.

2. Решаются системы алгебраических и дифференциальных уравнений (17) и (18) для определения матриц $K$ и $W(t)$ на отрезке $\left[t_{0}, T\right]$.

3. Задаются условия $y\left(t_{0}\right)=y_{0}$, и вычисляется $q\left(t_{0}\right)=W^{-1}\left(t_{0}\right) y\left(t_{0}\right)$.

4. Интегрируются на отрезке $\left[t_{0}, T\right]$ системы дифференциальных уравнений (20), (22) при начальных условиях $y\left(t_{0}\right)=y_{0}, \quad q\left(t_{0}\right)=W^{-1}\left(t_{0}\right) y\left(t_{0}\right)$.

\section{2. Численные эксперименты}

Рассматривается задача оптимального управления для экономической модели объекта управления, состоящего из трех секторов: $i=0$ (материальный сектор), $i=1$ (фондосоздающий сектор), $i=2$ (потребительский сектор), математическая модель включает $[15,16]$ :

а) три дифференциальных уравнения, описывающих динамику фондовооруженностей:

$$
\dot{k}_{i}=-\lambda_{i} k_{i}+\frac{s_{i}}{\theta_{i}} x_{1}, \quad k_{i}(0)=k_{i}^{0}, \lambda_{i}>0, \quad(i=0,1,2) ;
$$

b) три функции удельного выпуска типа Кобба-Дугласа:

$$
x_{i}=\theta_{i} A_{i} k_{i}^{\alpha_{i}}, \quad A_{i}>0, \quad 0<\alpha_{i}<1 \quad(i=0,1,2),
$$

с) три балансовых соотношения:

$$
\begin{gathered}
s_{0}+s_{1}+s_{2}=1, \quad s_{0} \geq 0, \quad s_{1} \geq 0, \quad s_{2} \geq 0, \\
\theta_{0}+\theta_{1}+\theta_{2}=1, \quad \theta_{0} \geq 0, \quad \theta_{1} \geq 0, \quad \theta_{2} \geq 0, \\
\left(1-\beta_{0}\right) x_{0}=\beta_{1} x_{1}+\beta_{2} x_{2}, \quad \beta_{0} \geq 0, \quad \beta_{1} \geq 0, \quad \beta_{2} \geq 0 .
\end{gathered}
$$

Здесь состояние экономической системы (фондовооруженность) описывается вектором $\left(k_{0}, k_{1}, k_{2}\right)$, а $\left(s_{0}, s_{1}, s_{2}, \theta_{0}, \theta_{1}, \theta_{2}\right)$ - вектор управлений, $\left(s_{0}, s_{1}, s_{2}\right)$ - доли секторов в распределении инвестиционных ресурсов, $\left(\theta_{0}, \theta_{1}, \theta_{2}\right)$ - доли секторов в распределении трудовых ресурсов; $x_{i}$ - удельный выпуск продукции в соответствующем секторе; $\beta_{i}$ - прямые материальные затраты в $i$-ом секторе; $i=0,1,2$. Начальное состояние системы равно $\left(k_{0}^{0}, k_{1}^{0}, k_{2}^{0}\right)$, где $k_{i}^{0}=k_{i}(0)$. Для решения задачи перевода нелинейной системы из начального состояния в желаемое состояние на отрезке времени $[0, T]$. В качестве желаемого конечного состояния $\left(k_{0}^{s}, k_{1}^{s}, k_{2}^{s}\right)$ используется состояние равновесия системы, которое определено в работе [12] в следующем виде:

$$
k_{0}^{s}=\frac{s_{0} \theta_{1} A_{1}\left(k_{1}^{s}\right)^{\alpha_{1}}}{\lambda_{0} \theta_{0}}, \quad k_{1}^{s}=\left(\frac{s_{1} A_{1}}{\lambda_{1}}\right)^{\frac{1}{1-\alpha_{1}}}, \quad k_{2}^{s}=\frac{s_{2} \theta_{1} A_{1}\left(k_{1}^{s}\right)^{\alpha_{1}}}{\lambda_{2} \theta_{2}} .
$$

Значения фондовооруженностей $k_{i}^{s},(i=0,1,2)$ в стационарном состоянии (39) зависят от управлений $\left(s_{0}, s_{1}, s_{2}, \theta_{0}, \theta_{1}, \theta_{2}\right)$, для которых в работе [12] получены стационарные значения $\left(s_{0}^{s}, s_{1}^{s}, s_{2}^{s}, \theta_{0}^{s}, \theta_{1}^{s}, \theta_{2}^{s}\right)$. Приведем теперь математическую модель объекта управления (34) к виду (1) и запишем в виде системы

$$
\dot{y}(t)=A y(t)+B(y) u(t)+D(y) v^{s} \quad y\left(t_{0}\right)=y_{0}, \quad t \in\left[t_{0}, T\right] .
$$


используя следующие обозначения $y_{1}=k_{1}-k_{1}^{s}, \quad y_{2}=k_{2}-k_{2}^{s}, \quad y_{3}=k_{0}-k_{0}^{s}, \quad u_{1}=s_{1}-v_{1}^{s}$,

$$
\begin{aligned}
& u_{2}=\frac{s_{2} \theta_{1}}{\theta_{2}}-v_{2}^{s}, \quad u_{3}=\frac{s_{0} \theta_{1}}{\theta_{0}}-v_{3}^{s}, \quad v_{1}^{s}=s_{1}^{s}, \quad s_{2}^{s} \theta_{1}^{s} / \theta_{2}^{s}=v_{2}^{s}, \quad s_{0}^{s} \theta_{1}^{s} / \theta_{0}^{s}=v_{3}^{s}, \\
& f_{1}\left(y_{1}\right)=\left(y_{1}+k_{1}^{s}\right)^{\alpha_{1}}, \quad f_{2}\left(y_{2}\right)=\left(y_{2}+k_{2}^{s}\right)^{\alpha_{2}}, \quad f_{3}\left(y_{3}\right)=\left(y_{3}+k_{0}^{s}\right)^{\alpha_{0}}, \\
& A=\left(\begin{array}{ccc}
-\lambda_{1}+b \alpha_{1}\left(k_{1}^{s}\right)^{\alpha_{1}-1} v_{1}^{s} & 0 & 0 \\
b \alpha_{1}\left(k_{1}^{s}\right)^{\alpha_{1}-1} v_{2}^{s} & -\lambda_{2} & 0 \\
b \alpha_{1}\left(k_{1}^{s}\right)^{\alpha_{1}-1} v_{3}^{s} & 0 & -\lambda_{0}
\end{array}\right), \quad B=\left(\begin{array}{ccc}
b\left(y_{1}+k_{1}^{s}\right)^{\alpha_{1}} & 0 & 0 \\
0 & b\left(y_{1}+k_{1}^{s}\right)^{\alpha_{1}} & 0 \\
0 & 0 & b\left(y_{1}+k_{1}^{s}\right)^{\alpha_{1}}
\end{array}\right) \text {, } \\
& B(0)=B_{s}, \quad D(y)=\left(\begin{array}{ccc}
d\left(y_{1}\right) & 0 & 0 \\
0 & d\left(y_{1}\right) & 0 \\
0 & 0 & d\left(y_{1}\right)
\end{array}\right), \quad D\left(y_{1}\right)=b\left(y_{1}+k_{1}^{s}\right)^{\alpha_{1}}-\left(k_{1}^{s}\right)^{\alpha_{1}}-\alpha_{1}\left(k_{1}^{s}\right)^{\alpha_{1}-1} y_{1} .
\end{aligned}
$$

Здесь постоянные значения $k^{s}$ и $v^{s}$ определяются в (40) и для них имеет место алгебраическая связь

$$
A k^{s}+B D\left(k^{s}\right) v^{s}=0 .
$$

Для применения алгоритма решения задачи в примере дополнительно введем функции

$$
\begin{gathered}
f_{1}\left(y_{1}\right)=\left(y_{1}+k_{1}^{s}\right)^{\alpha_{1}}, \quad f_{2}\left(y_{2}\right)=\left(y_{2}+k_{2}^{s}\right)^{\alpha_{2}}, \quad f_{3}\left(y_{3}\right)=\left(y_{3}+k_{3}^{s}\right)^{\alpha_{3}}, \\
\xi=\frac{\beta_{1} A_{1} f_{1}\left(y_{1}\right)+\beta_{2} A_{2} f_{2}\left(y_{2}\right)\left(1-u_{1}-v_{1}^{s}\right) /\left(u_{2}+v_{2}^{s}\right)}{\left(1-\beta_{0}\right) A_{0} f_{3}\left(y_{3}\right)\left(1-u_{1}-v_{1}^{s}\right) /\left(u_{3}+v_{3}^{s}\right)+\beta_{2} A_{2} f_{2}\left(y_{2}\right)\left(1-u_{1}-v_{1}^{s}\right) /\left(u_{2}+v_{2}^{s}\right)},
\end{gathered}
$$

которые обеспечивают выполнение условия (38); при этом инвестиционные ресурсы

$$
s_{1}=u_{1}+v_{1}^{s}, \quad s_{2}=(1-\xi)\left(1-u_{1}-v_{1}^{s}\right), \quad s_{0}=\xi\left(1-u_{1}-v_{1}^{s}\right)
$$

обеспечивают выполнение условия (36); а трудовые ресурсы

$$
\theta_{1}=\frac{1}{1+\left(s_{0}\right) /\left(u_{3}+v_{3}^{s}\right)+\left(s_{2}\right) /\left(u_{2}+v_{2}^{s}\right)}, \quad \theta_{2}=\frac{(1-\xi)\left(1-s_{1}\right) \theta_{1}}{u_{2}+v_{2}^{s}}, \quad \theta_{0}=\frac{\xi\left(1-s_{1}\right) \theta_{1}}{u_{3}+v_{3}^{s}}
$$

обеспечивают выполнение условия (37).

Были проведены численные расчеты на компьютере при следующих значениях параметров:

$$
\begin{gathered}
\alpha_{0}=0,46 ; \quad \alpha_{1}=0,68 ; \quad \alpha_{2}=0,49 ; \quad \beta_{0}=0,39 ; \quad \beta_{1}=0,29 ; \quad \beta_{2}=0,52 ; \\
\lambda_{i}=0,05, i=0,1,2 ; \quad A_{0}=6,19 ; \quad A_{1}=1,35 ; \quad A_{2}=2,71 ; \\
s_{0}^{s}=0,2763 ; \quad s_{1}^{s}=0,4476 ; \quad s_{2}^{s}=0,2761 ; \quad \theta_{0}^{s}=0,3944 ; \quad \theta_{1}^{s}=0,2562 ; \quad \theta_{2}^{s}=0,3494 ; \\
k_{0}^{s}=966,4430 ; \quad k_{1}^{s}=2410,1455 ; \quad k_{2}^{s}=1090,1238 ;
\end{gathered}
$$

Начальные условия задаются следующие $y\left(t_{0}\right)=(-700,-300,300)^{\prime}$, а матрицы $R, Q_{1}, K$ имеют вид $R=\left(\begin{array}{ccc}350 & 0 & 0 \\ 0 & 70 & 0 \\ 0 & 0 & 55\end{array}\right), Q_{1}=\left(\begin{array}{ccc}49 \cdot 10^{-4} & 0 & 0 \\ 0 & 9 \cdot 10^{-4} & 0 \\ 0 & 0 & 9 \cdot 10^{-4}\end{array}\right), K=\left(\begin{array}{ccc}0,4598 \cdot 10^{-2} & 0,6894 \cdot 10^{-5} & 0,5129 \cdot 10^{-5} \\ 0,6894 \cdot 10^{-5} & 0,8888 \cdot 10^{-3} & -3,5598 \cdot 10^{-9} \\ 0,5129 \cdot 10^{-5} & -3,5598 \cdot 10^{-9} & 0,7926 \cdot 10^{-3}\end{array}\right)$.

Результаты расчетов состояния системы представлены на рисунке 1(а). Из рисунка 1(б) видно, что оптимальные управления не выходят за пределы области $U$, определяемой ограничениями $-0.3477 \leq u_{1} \leq 0.4523, \quad-0.1024 \leq u_{2} \leq 0.6976, \quad-0.07945 \leq u_{3} \leq 0.7205$. 


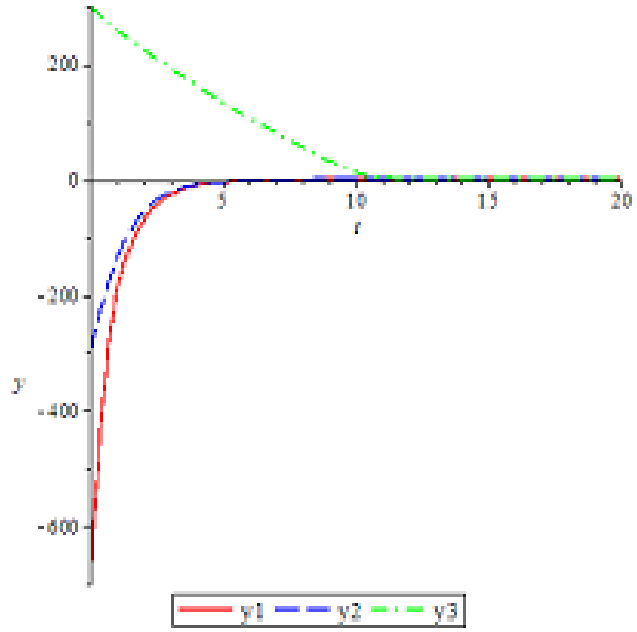

(a)

Fig. 1. Graphs of trajectories $y(t)(a)$ and the optimal control $u(t)(b)$

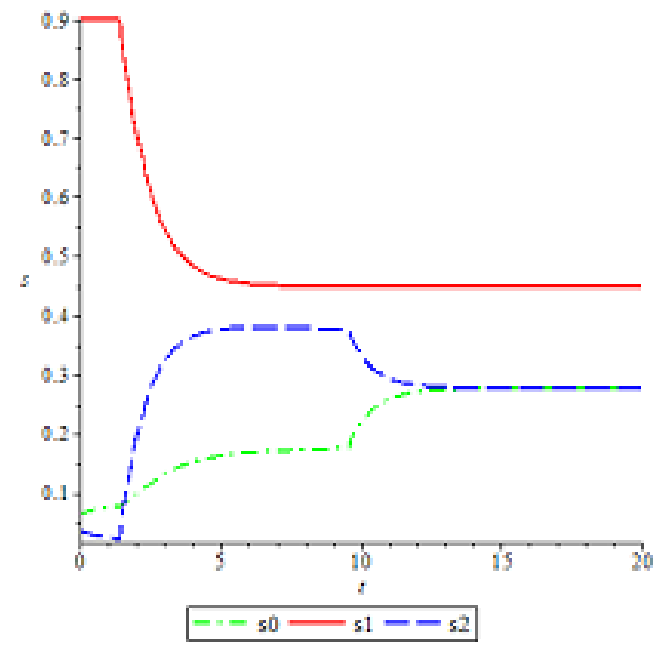

(a)

Fig. 2. Graphs of the distribution of investment (a) and labor resources (b)

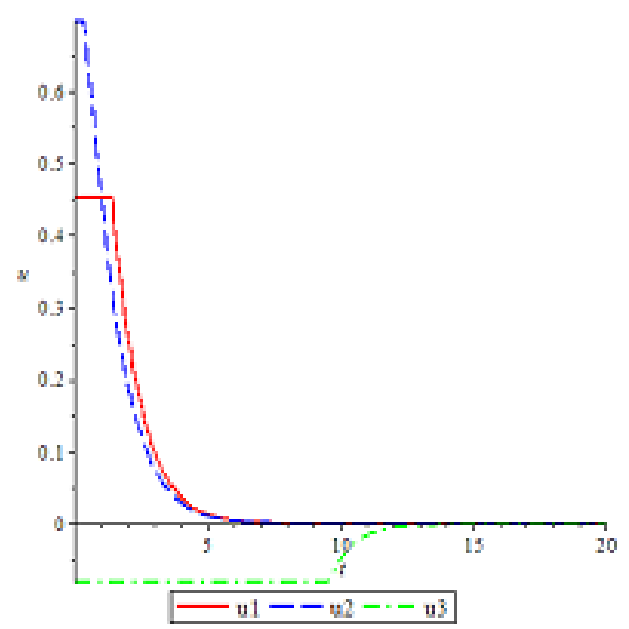

(b)

Рис. 1. Графики траекторий $y(t)(a)$ и оптимального управления $u(t)$ (b)

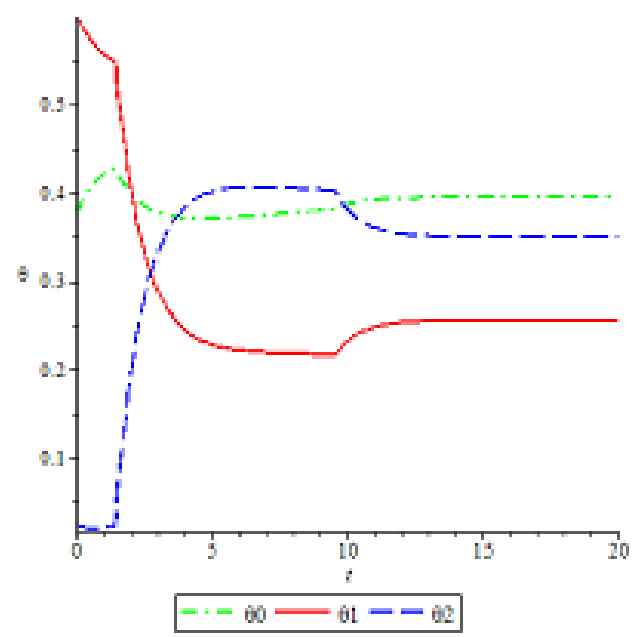

(b)

Рис. 2. Графики распределения инвестиций (а) и трудовых ресурсов (b)

Здесь все компоненты управления $u_{1}(t), u_{2}(t)$ и $u_{3}(t)$ лежат на границе области $U$ на отрезке $\left[0, t_{1}\right],\left[0, t_{2}\right]$ и $\left[0, t_{3}\right]$ соответственно, затем при $t \in\left(t_{1}, T\right], t \in\left(t_{2}, T\right]$ и $t \in\left(t_{3}, T\right]$ заходят внутрь области $U$. Переключение управлений происходит в момент времени $t_{1}=1,439$ для компонента $u_{1}(t)$, а для $u_{2}(t)$ при $t_{2}=0,4$, для $u_{3}(t)$ при $t_{3}=9,785 . y_{1}(T)=-4.8692 \cdot 10^{-6} ; \quad y_{2}(T)=-1.7315$. $10^{-6} ; \quad y_{3}(T)=0.229 \cdot 10^{-3}$, и оптимальные значения управлений в конечный момент времени при $T=20: u_{1}(T)=2.2873 \cdot 10^{-8} ; \quad u_{2}(T)=6.5404 \cdot 10^{-9} ; \quad u_{3}(T)=-3.4357 \cdot 10^{-7}$. На рисунке 2 показаны изменения ресурсов, которые удовлетворяют балансовым соотношениям (36), (37). Значения инвестиций $\left(s_{0}(t), s_{1}(t), s_{2}(t)\right)$ и трудовых ресурсов $\left(\theta_{0}(t), \theta_{1}(t), \theta_{2}(t)\right)$ в конечный момент времени при $T=20$ стремятся к стационарному состоянию. 


\section{Заключение}

Для частного класса нелинейных управляемых систем на конечном интервале показывается возможность применения подхода SDRE при построении обратной связи на задаче с ограничениями на управление, применяя принцип расширения, предложенного В.Ф. Кротовым и развитый В.И. Гурманом, и при этом предлагается эффективный алгоритм, не требующий многократного интегрирования дифференциального уравнения Риккати с коэффициентами, зависящими от состояния.

Также приведены численные эксперименты, иллюстрирующие предлагаемый алгоритм построения оптимального синтеза при наличии ограничений на управление в виде замкнутых неравенств на примере трехсекторной нелинейной экономической системы.

\section{References}

[1] C. Mracek and J. Cloutier, "Control designs for the nonlinear benchmark problem via the state-dependent Riccati equation method”, International fournal of Robust and Nonlinear Control, vol. 8, no. 4-5, pp. 401-433, 1998.

[2] J. R. Cloutier and D. T. Stansbery, "The Capabilities and Art of State-Dependent Riccati Equation-Based Design”, in Proceedings of the American Control Conference, vol. 1, IEEE, Piscataway, May, 2002, pp. 86-91.

[3] V. Afanas'ev and P. Orlov, "Suboptimal control of a nonlinear object linearized by feedback", Bulleten RAS. Control theory and systems, no. 3, pp. 13-22, 2011, (In Russian).

[4] T. Cimen, "State-dependent Riccati Equation (SDRE) control: A Survey", IFAC Proceedings Volumes, vol. 41, no. 2, pp. 3761-3775, 2008.

[5] A. Heydari and S. N. Balakrishnan, "Path Planning Using a Novel Finite Horizon Suboptimal Controller", Journal if Guidance, Control and Dynamics, vol. 36, no. 4, pp. 1210-1214, 2013.

[6] A. Heydari and S. N. Balakrishnan, "Approximate closed-form solutions to finite-horizon optimal control of nonlinear systems", in American Control Conference (ACC), IEEE, 2012, pp. 2657-2662.

[7] V. F. Krotov and V. Gurman, Methods and Problems of Optimal Control. Nauka, Moscow, 1973.

[8] V. Gurman, The principle of expansion in control tasks. Moscow "Science" Main Editing of Physical-Mathematical Literature, 1985, (In Russian).

[9] M. Dmitriev, Z. Murzabekov, D. Makarov, and G. Mirzakhmedova, "SDRE based stabilization of the affine control system with the stationary linear part”, in 23rd International Conference on System Theory, Control and Computing, 2019, pp. 739-743.

[10] S. Aipanov and Z. Murzabekov, "Analytical solution of a linear quadratic optimal control problem with control value constraints”, fournal of Computer and Systems Sciences International, vol. 1, no. 53, pp. 84-91, 2014.

[11] Z. Murzabekov, "The synthesis of the proportional-differential regulators for the systems with fixed ends of trajectories under two-sided constraints on control values”, Asian fournal of Control, vol. 2, no. 18 , pp. 494-501, 2016.

[12] Z. Murzabekov, M. Milosz, and K. Tussupova, "The optimal control problem with fixed-end trajectories for a three-sector economic model of a cluster", in 10th International scientific conferences on research and applications in the field of intelligent information and database systems, ACIIDS, Dong Hoi City, 2018, pp. 382-391. 
[13] Z. Murzabekov, M. Milosz, and K. Tussupova, "Modeling and optimization of the production cluster", in Proceedings of 36th International Conference on Information Systems and Architecture and Technology - ISAT-2015 / Part II, Advances in Intelligent Systems and Computing. - Karpacz, 2016, pp. 99-108.

[14] M. Dmitriev and D. A. Makarov, "A weak non-linear regulator in a weakly non-linear control system with efficiency”, Proceedings of the ISA RAS, vol. 4, no. 64, pp. 53-58, 2014, (In Russian).

[15] V. A. Kolemaev, "Optimal balanced space of the open three-sector economy", Applied econometrics, vol. 3, no. 11, pp. 15-42, 2008, (In Russian).

[16] S. M. Aseev, K. Besov, and A. Kryazhimsky, "Optimal control problems on infinite time management in economics", Advances in mathematical sciences, vol. 404, no. 2, pp. 3-64, 2012, (In Russian). 